\title{
Auditoría de Gestión al área de Créditos y Cobranzas en Instituciones Religiosas sin Fines de Lucro
}

\section{Management Audit to the area of Credits and Collection in Non-Profit Religious Institutions}

DOI: $10.46932 / \mathrm{sfjdv} 3 \mathrm{n} 1-040$

Received in: Dec 30st, 2021

Accepted in: Jan 1th, 2022

\author{
Erick E. Obaco Bastidas \\ Pontificia Universidad Católica del Ecuador, Santo Domingo de los Tsáchilas \\ Av. Chone Km 2, Santo Domingo 230203 \\ E-mail: eeobacob@pucesd.edu.ec \\ Katherine M. Delgado Montalván \\ Pontificia Universidad Católica del Ecuador, Santo Domingo de los Tsáchilas \\ Av. Chone Km 2, Santo Domingo 230203 \\ E-mail: kmdelgadom@pucesd.edu.ec \\ Ximena L. García Zambrano \\ $\mathrm{Mg}$ \\ Pontificia Universidad Católica del Ecuador, Santo Domingo de los Tsáchilas \\ Av. Chone Km 2, Santo Domingo 230203 \\ E-mail: xlgarciaz@pucesd.edu.ec
}

\section{RESUMEN}

El área de créditos y cobranzas es indispensable en toda institución, su dirección, manejo y organización es un factor clave para el éxito de la misma, resaltando la importancia de la existencia de un manual de procedimientos que detalle de forma específica la acción a seguir al momento de conceder un crédito para evitar altercados futuros con los clientes y una metodología de cobro efectiva que garantice en la mayoría de las situaciones una recuperación total de la cartera, adicionalmente del respectivo control y seguimiento en los procedimientos que son indicadores de que la labor se realiza de forma satisfactoria y el sistema actual funciona, trabajo realizado aplicando una auditoría específicamente de gestión, en donde cada etapa de su proceder fue planificada con anterioridad, recopilando información, identificando hallazgos, contrastando información, realizando papeles de trabajo y presentando los resultados construidos en bases sólidas en el informe final de auditoría por medio de opiniones.

Palabras clave: Concesión, recuperación, cartera, crédito, auditoría de gestión, cobranzas, cuentas por cobrar.

\begin{abstract}
The area of credits and collections is essential in every institution, its direction, management and organization is a key factor for its success, highlighting the importance of the existence of a procedures manual that specifically details the action to be followed when moment of granting a loan to avoid future altercations with clients and an effective collection methodology that guarantees in most situations a total recovery of the portfolio, in addition to the respective control and monitoring in the procedures that are indicators that the work is performed satisfactorily and the current system works, work carried out by
\end{abstract}


applying a specifically management audit, where each stage of its procedure was previously planned, collecting information, identifying findings, contrasting information, performing work papers and presenting the results built in solid foundations in the final audit report through of opinions.

Keywords: Concession, recovery, portfolio, credit, management audit, collections, accounts receivable.

\section{INTRODUCCIÓN}

El área de créditos y cobranzas denominada comúnmente como "cuentas por cobrar" es esencial en el manejo de toda organización, debe ser entendida como un factor clave para el éxito, cuya gestión esta intrínsecamente relacionada con el nivel de control que la empresa posee sobre clientes que presentan deudas, así como la eficiencia en su sistema de cobros; los saldos a recuperar son efectivo propio de la organización los cuales están bajo dominio del cliente y es imprescindible que regrese a la entidad, para solventar cualquier tipo de requerimiento o eventualidad que pueda acontecer, resaltando la importancia de que una gestión adecuada favorecerá al cumplimiento de los objetivos empresariales.

Es cierto que la auditoria es un procedimeinto que proporciona certeza, pues los resultados obtenidos a través de la misma son característicamente transparentes, más la calidad de los hallazgos está estrictamente ligada a la cantidad y veracidad de la información proporcionada por el organismo, ente o institución que se encuentre como objeto del examen, la cual en su mayoria es el limitante principal de la auditoria pues al no contar con los datos necesarios el análisis realizado es superficial, haciendo posible la omisión de ciertas áreas críticas o aspectos importantes los cuales requieren atención, creando desconfianza en ciertas situaciones al no poder determinar los niveles de riesgo o incorrección material.

El fenómeno desconfianza se puede analizar basándose en el trabajo de Martínez et al,. (2010) donde señala que el origen de la crisis de confianza es atribuible de forma directa al caso Enron-Andersen, dejando en evidencia al sistema contable anglosajon orillando a paises pertenecientes a Ibero America a fortalecer el marco regulador y normativo de la auditoría, dando lugar a la creación de un plan el cual sería dirigido bajo orientación de las NIC, NIIF y las NIAS. En Ecuador según Prado et al., (2019) en el periodo 2018, Esmeraldas posee el mas alto grado de morosidad con 6,8\% seguida por Napo con 6,5\%, en lo que respecta a Santo Domingo de los Tsáchilas esta ocupa el décimo puesto con un nivel de morosidad equivalente a $3,3 \%$.

El área no gubernamental sin afan al lucro ha ganado gran influencia en el ámbito colectivo y económico, no solo a una escala local pues su fuerza inclusive trasciende internacionalmente pues se especula que su gestión supera al gobierno en lo que respecta a proporcionar servicios sociales, prestados a través de diversos programas para lo que es evidente una necesidad de financiamiento a largo plazo que garantice la sostenibilidad de proyecto, es decir rentabilidad económica, obligando a las empresas no 
lucrativas a explotar su ingenio, formulando estrategias que les permitan continuar con las metas propuestas en beneficio de la comunidad (Betancur-Marín \& Acosta-Santamaría, 2014).

La necesidad del efectivo, fondos, dinero o financiamiento es un argumento presente entre las entidades sin afan al lucro, necesidad que crecer con un fuerza descomunal pues los directivos se enfocan en la creación de los programas de obra social mas no en su patrocinio, esta necesidad es mas evidente en periodos de crisis económica dado que surgen las siguientes interrogantes, ¿Cuál es la cantidad necesaria? ¿Cómo la podemos obtener? ¿Es suficiente lo recaudado?, las diferentes interrogantes generan alboroto,favoreciendo a la desorganiganición entorpeciendo el manejo y dirección efectiva de los fondos provocando lo que se conoce como recortes o inclusive la eliminación de los proyectos mas prometedores (Landes-Foster et al., 2021).

Para esta investigación se tomó como objeto de estudio a la empresa Diócesis de Santo Domingo en Ecuador debido a que encaja en el perfil de la temática central, es una entidad religiosa sin afan de lucro, cuya orientación primaria es promover la labor social a través de sus diversos programas, posee diversas fuentes de financiamiento pero el área a investigar es la de créditos y cobranzas dado que se ha identificado que la institución no posee definidas de forma escrita políticas, procedimientos e inclusive no existe un departamento específico que se encargue de la tarea.

\section{REVISIÓN DE LA LITERATURA}

\subsection{AUDITORÍA}

\subsubsection{Origen y antecedentes internacionales de la auditoría}

El mérito del nacimiento de la auditoría se lo atribuye Gran Bretaña, con el comienzo de la Revolución Industrial y la bancarrota de varias empresas, surgió como una metodología que garantizaba la fiabilidad de la información financiera, aunque varios expertos afirman que su origen es más antiguo pues existen vestigios de la práctica de la auditoría aplicada a través de rústicos sistemas de supervisión, aplicados con el propósito de evitar fraudes, timos y estafas en las relaciones comerciales mantenidas. Esta práctica según respaldan varias investigaciones era exigida e implementada por las altas monarquías y estratos sociales altos, anticipándose a cualquier tipo de desfalco o hurto por parte de sus trabajadores (Tapia et al., 2019).

\subsection{ASPECTOS GENERALES DE LA AUDITORÍA:}

La contaduría y la auditoría son áreas cambiantes que se encuentran en constante desarrollo, el cual es directamente proporcional al nivel de evolución humana; entre los siglos XIX y XX su aplicación presentaba un alto grado de complejidad al no existir un marco reglamentario universal (NIIF), surgiendo la necesidad de homogeneizar sus procesos, necesidad que se pudo evidenciar en el año 2000, en donde 
varios contadores y auditores perdieron su credibilidad por una serie de escándalos financieros ocurridos en EE. UU.; obligando a las empresas a implementar un riguroso sistema de control con la finalidad de recuperar el prestigio y confianza en la profesión (Tapia et al., 2019).

\subsection{AUDITORÍA DEFINICIÓN GENÉRICA:}

Actividad orientada a la corroboración, revisión y control de que las actividades realizadas dentro de una organización o área de supervisión se ejecutaron respetando la planificación previamente establecida, políticas empresariales y principalmente los principios de contabilidad generalmente aceptados (Label et al., 2016).

\subsection{AUDITOR DEFINICIÓN GENÉRICA:}

Término utilizado para referirse al socio de una firma auditora el cual posee facultad de realizar un trabajo de auditoría de cualquier naturaleza en una organización determinada bajo los principios de contabilidad generalmente aceptados con total ética, profesionalidad e imparcialidad desde el inicio hasta su culminación (Label et al., 2016).

\subsection{TIPOS DE AUDITORÍA}

Biler-Sofía (2017) señalan que la existencia de tantas variantes de la auditoría es atribuible a las difrentes necesidades que se presentan en las organizaciones, actualmente casi todo se puede auditar, se crean perspectivas distintas proporcionando diferentes soluciones a los que talvez con ciertos tipos de auditoría no se pueda llegar. Como se menciona anteriormente existen diferentes variantes de la auditoría pero todas comparten un procedimiento similar, los cuales se centran en una minuciosa supervisión e identificación de los posibles inconvenientes además de su respectiva solución.

\subsection{DEFINICIÓN AUDITORÍA DE GESTIÓN:}

Se refiere al proceso de recopilación de información realizado en el transcurso de la auditoría, cuyo propósito es proporcionar opiniones construidas bajo hechos sólidos, aplicando un juicio profesional transparente que resalte la imparcialidad, referente a la situación actual de la organización es decir el grado de eficiencia y eficacia con el que desempeñan sus operaciones (Alcívar-Cedeño et al., 2016).

\subsection{FASES DE LA AUDITORÍA DE GESTIÓN}

Fase de exploración y examen preliminar: Fase inicial del proceso de auditoría la cual consiste en una observación superficial generalmente de documentación de periodos anteriores que actuarán como guía en la identificación de áreas críticas, también puede acontecer que los inconvenientes sean identificados al iniciar la auditoría (Arias-Gonzáles, 2018). 
Fase de planificación de la auditoría de gestión: Segunda fase del proceso y consiste básicamente en la estructuración del plan operativo anual de auditoría, el cual es intrínsecamente realizado para la institución a auditar, se contrastará información tanto de ejercicios anteriores como el actual y su rasgo más notable es su flexibilidad debido a que este puede ser modificado en cualquier punto del proceso si la situación así lo requiera (Cubero-Abril, 2019).

Fase de ejecución auditoría de gestión: En esta fase el auditor aplica diferentes evaluaciones a las evidencias obtenidas en la fase de planificación las cuales deben estar respaldadas por la documentación respectiva, con el propósito de encontrar hallazgos que serán presentados a los directivos de la empresa en el respectivo informe de auditoría (Cubero-Abril, 2019).

Papeles de trabajo: Evidencias físicas recolectadas por el auditor en el transcurso de la auditoría que indican que la metodología fue seguida según las pautas detalladas en el plan operacional de auditoría. Se utilizan como fundamento en las opiniones construidas por el auditor (Campos-Muñiz et al. 2018).

Comunicación de los resultados, Informe de auditoría: Esta fase se refiere al resultado final del proceso de auditoría, en ella se presentarán todos los hallazgos realizados por parte del auditor en el transcurso de la auditoría sin importar que estos sean positivos o negativos, los mismos que son presentados ante la junta de socios y otras partes interesadas. Este procedimiento se realiza con el fin de dar a conocer a la empresa las deficiencias encontradas y la respectiva solución aportando valor a la empresa, promoviendo su crecimiento (Cubero-Abril, 2019).

Seguimiento: Última fase del proceso de una auditoría de gestión tiene lugar después de la presentación del informe final de auditoría, se realiza con el propósito de evaluar si la gerencia o la administración aplican eficientemente las recomendaciones redactadas en el informe de auditoría, estas evaluaciones se realizan según el cronograma establecido en el POA (Cubero-Abril, 2019).

\subsection{CRÉDITOS Y COBRANZAS:}

El periodo de conveniencia donde es favorable para un cliente obtener un bien o producto de inmediato y cancelarlo en una fecha futura se denomina crédito, metodología actual que favorece el movimiento de los inventarios y está respaldada en el convenio empresa-cliente pactado anteriormente. Su finalidad es ofrecer diferentes facilidades a los clientes para lo que es imprescindible un sistema sólido que garantice la lealtad de los usuarios creando vínculos fuertes además de métodos e instrumentos que garanticen la recuperación del crédito. La concesión de un crédito no es un procedimiento empírico, es de gran importancia que la empresa cuente con lineamientos definidos para analizar la situación del solicitante considerando si es posible negar o cumplir la solicitud (Romo-Veloz, 2016). 


\subsection{ADMINISTRACIÓN DEL CRÉDITO:}

Trabaja con un control adecuado sobre los procedimientos crediticios y administrativos, proporcionando varias facilidades que hacen más sencillo aplicar un correcto control de los rubros que están en proceso de recuperación y cancelación, anticipando posibles escenarios, como por ejemplo el incumplimiento de las cancelaciones de créditos otorgados; este procedimiento es aplicado con el propósito de identificar las posibles alternativas o medidas a seguir si existiera incumplimiento ya sea parcial o total de la cancelación de los saldos por parte de los clientes (Ulloa-Zuñiga, 2020).

\subsection{POLÍTICAS DE CRÉDITO Y COBRANZA:}

Normativa de actuación determinada según la necesidad de la organización, empleadas como estrategias buscando contrarrestar riesgos, específicamente en el ámbito financiero, determinando estándares o parámetros efectivos para conceder un crédito al cliente, así como el respectivo proceso de recuperación (Romo-Veloz, 2016).

\subsection{DEFINICIÓN DE CRÉDITO:}

Procedimiento o acción de carácter financiero en donde intervienen generalmente dos individuos, el que proporciona el efectivo solicitado se denomina acreedor y puede ser una persona natural o jurídica, el deudor quien es el interesado y además se compromete a saldar dicho préstamo de forma gradual en el periodo de tiempo determinado al inicio del convenio, recalcando que el mismo se estableció por acuerdo entre ambas partes (Montes de Oca, 2015).

\subsection{DEFINICIÓN DE COBRANZA:}

Procedimiento formal que consiste en la recuperación de los créditos otorgados previamente por parte de la institución o empresa al cliente, el mismo que requiere de una estricta sistematización que asegure de forma total la restitución de los saldos vencidos de la organización, resaltando la importancia de la existencia de un manual específicamente detallado para el área de créditos y cobranzas (Lopez, 2016).

\subsection{CARTERA VENCIDA:}

Surge en el momento que el cliente no cumple con la obligación pactada en el periodo determinado, dando lugar a la mora término que es sinónimo de incumplimiento a la normativa establecida pasando a denominarse como cuentas incobrables, efectivo que, si no es recuperado, aunque sea de forma parcial afectará a la empresa en el ámbito económico afectando su liquidez (Cuenca-Zapata, 2017). 


\section{PREGUNTAS DE INVESTIGACIÓN}

Todos los acontecimientos y datos presentados anteriormente condijeron a la formulación de la siguiente problematica: ¿Qué beneficios tendría la Diócesis de Santo Domingo al realizar una auditoría al proceso de concesión y recuperación de la cartera?, la cual se segmento en las siguientes preguntas específicas:

1. ¿Cómo conocer las actividades y procedimientos de importancia en el proceso concesión y recuperación de cartera?

2. ¿Cómo determinar la metodología y actividades a realizar en el proceso de auditoría de gestión?

3. ¿Cómo beneficia a la ejecución del proceso de auditoría de gestión un plan de trabajo definido?

4. ¿Cómo presentar a la empresa los hallazgos realizados dentro del proceso de auditoría al área de créditos y cobranzas?

\section{OBJETIVOS DE INVESTIGACIÓN}

\section{Objetivo general:}

Realizar una auditoría de gestión al proceso de concesión y recuperación de cartera de la Diócesis de Santo Domingo para mejorar los procedimientos del departamento de créditos y cobranzas.

\section{Objetivos específicos:}

1. Levantar información acerca del proceso de concesión y recuperación de cartera para estructurar una metodología de trabajo efectiva.

2. Elaborar los programas de trabajo para realizar el proceso de auditoría y determinar el nivel de riesgo y confianza de los procesos a auditar

3. Determinar los hallazgos mediante la ejecución de las actividades propuestas de los programas de trabajo.

4. Elaborar un informe de resultados de la auditoría para expresar opiniones y recomendaciones de las deficiencias encontradas en el proceso de créditos y cobranzas.

\section{METODOLOGIA DE LA INVESTIGACIÓN}

\subsection{ENFOQUE, TIPO DE INVESTIGACIÓN Y DISEÑO}

La naturaleza de la presente investigación es netamente descriptiva, debido a que se identificaron las características principales de los procesos de concesión y recuperación de la cartera, cuyos resultados pueden ser interpretados ya sea a través de un análisis tanto cualitativo como cuantitativo. SánchezCarlessi et al. (2018) señala que un estudio de carácter descriptivo, identifica la situación presente o características actuales mas representativas del acontecimiento o fenómeno el cual es objeto de estudio.Referente al enfoque para esta investigación se utilizó el cuantitativo, que hace alusión a los datos 
de índole númerica que son interpretados en diferentes niveles de medición además de técnicas descriptivas e inferenciales; en lo que respecta al enfoque o análisis cualitativo, se refiere al estudio de datos e información no numéricos que consideran diferentes niveles de cuantificación además de la aplicación de técnicas netamente descriptivas e inferenciales (Sánchez-Carlessi et al., 2018).

Considerando las premisas descritas anteriormente se puede deducir que el enfoque utilizado en esta investigación es mixto, se analizaron los niveles de eficiencia y eficacia de los procedimientos de concesión y recuperación de cartera, a través de varios indicadores y otros diversos instrumentos de medición que facilitaron el análisis e interpretación de los datos obtenidos.

\subsection{POBLACIÓN OBJETO DE ESTUDIO}

Arias-Gómez et al., (2016) se refiere a población como la aglomeración de casos, individuos, sucesos cuyos rasgos específicos varian según el tipo de investigación que se esté llevando a cabo, estas características facilitan la identificación y selección de los candidatos óptimos que formarán parte de la muestra previamente definida. La DIÓCESIS de Santo Domingo cuenta con 24 empleados y 34 clientes, la población objeto de estudio de esta investigación son dos empleados, directamente relacionados con el proceso de concesión y recuperación de cartera (administradora y contadora interna) y los 34 clientes que conforman su cartera. El total de la población lo conforman 36 personas, no fue necesario aplicar un muestreo por la naturaleza de la población (finita), ya que se requirió la información específica que proporcionaron los empleados y los clientes que integran la cartera de la empresa.

\subsection{TÉCNICAS E INSTRUMENTOS DE RECOGIDA DE DATOS}

El cuestionario es una herramienta de recopilación de datos de naturaleza empírica que otorga la facultad de estandarizar y unificar el proceso de recopilación de información considerando la metodología y enfoque de la investigación (López-Roldan \& Fachelli, 2015). El instrumento de recogida de datos utilizado en el trabajo de investigación es el cuestionario que se elaboró en referencia a los lineamientos establecidos en el COSO I y II, el cual una vez pulido y finalizado fue aplicado a la población objeto de estudio, la misma que está integrada por los empleados de la DIÓCESIS de Santo Domingo que forman parte del proceso de créditos y cobranzas incluida la cartera de clientes.

La entrevista es una herramienta de recolección de datos, preferida por su facilidad de aplicación, ya que es un diálogo convencional que ocurre con la finalidad de reunir información relevante para la investigación (Díaz-Bravo et al., 2013). Se aplicó una entrevista de naturaleza cualitativa con el propósito de que la administración de la empresa detallara los procesos que se siguen en el departamento de cobranzas, los cuales fueron analizados a través de indicadores que identificaron las deficiencias, además de su nivel de eficiencia y eficacia. 


\subsection{OPERACIONALIZACIÓN DE LAS VARIABLES}

Tabla 1 Operacionalización de las Variables

\begin{tabular}{|c|c|c|}
\hline Variable & Dimensión & Indicador \\
\hline \multirow{7}{*}{ Auditoría de gestión (variable independiente) } & \multirow{2}{*}{ Nivel de riesgo } & \# Controles aplicados \\
\hline & & Total procedimientos \\
\hline & \multirow{2}{*}{ Eficiencia } & \# Controles evaluados \\
\hline & & Total controles \\
\hline & \multirow{2}{*}{$\begin{array}{l}\% \text { cumplimiento del } \\
\text { programa de auditoria }\end{array}$} & \# Actividades realizadas \\
\hline & & \#Actividad programadas \\
\hline & \multirow{2}{*}{ Nivel de confianza } & \# R. positivas \\
\hline \multirow{8}{*}{ Cuentas por cobrar (variable dependiente) } & & $\begin{array}{l}\text { Total respuestas } \\
\text { Ventas }\end{array}$ \\
\hline & Rotación de cartera & Cuentas por cobrar \\
\hline & \multirow{2}{*}{ Morosidad de la cartera } & Cuentas Incobrables \\
\hline & & Total cuentas por cobrar \\
\hline & \multirow{2}{*}{$\begin{array}{l}\text { Periodo de cobranza de la } \\
\text { Cartea }\end{array}$} & Días transcurridos \\
\hline & & Rotación de Cartera \\
\hline & \multirow{2}{*}{ Liquidez } & Activo Circulante \\
\hline & & Pasivo Corto plazo \\
\hline
\end{tabular}

Nota. Dimensión de las variables dependiente e independiente. Fuente: Elaboración propia.

\subsection{TÉCNICAS DE ANÁLISIS DE DATOS}

Quintanilla-Romero \& Trujillo-Caldero (2015) señala que las pruebas de control son herramientas que se utilizan para corroborar el cumplimiento de los procedimientos, proporcionando un nivel de confianza adecuado de que los procedimientos, acciones, políticas y objetivos se cumplen según lo previsto, en lo que respecta a las pruebas sustantivas se direccionan a la supervisión y análisis de los saldos en los estados financieros con la finalidad de asegurar la validez de la información contable.

Las técnicas para el análisis de los datos que se utilizaron en este estudio son las pruebas de cumplimiento y sustantivas con las que se verificaron si los procedimientos se realizaban según la empresa desee y la validez de la información contable. Adicionalmente se representaron en diferentes gráficos (circulares-anillos), los resultados de las herramientas de recogida de datos, en el cual cada respuesta fue representada por un color diferente para facilitar la compresión y al final de cada gráfico se redactó el análisis respectivo basado en los resultados.

\section{RESULTADOS}

\subsection{ANÁLISIS DE LOS INSTRUMENTOS APLICADOS A LOS ACTORES INTERNOS Y EXTERNOS}

Los datos obtenidos al realizar la entrevista a la administradora evidenciaron que la empresa estudiada es una organización religiosa sin fines de lucro, orientada a la labor social que es financiada a través del arriendo de bienes inmuebles y otras actividades. Su proceso de recuperación de cartera es ineficiente por falta de solidez en su estructura y los diferentes altercados que surgen con los clientes, considerando que al pactar el contrato notariado se detallan los requisitos. La supervisión a los plazos de 
vencimientos a través de las bases de datos son una de las medidas para evitar los contratiempos, el diálogo, el que, si no es efectivo, dará lugar al respectivo procedimiento legal, resaltado ausencia de rigurosidad en los procesos.

La entrevista realizada a la contadora indicó su tiempo de labor en la empresa (27 años), su rol dentro el proceso de cobranzas y la solidez del mismo, aunque presenta ciertas falencias, que se dan principalmente por inconvenientes con los arrendatarios, complicando la recuperación de la cartera en los plazos determinados. Se presentan informes periódicos que la administradora utiliza para analizar y tomar decisiones con respecto a los retrasos de los cánones de arriendo. Se concluyó con el reconocimiento de la importancia de una auditoría al proceso de cobranzas para garantizar su efectividad.

El cuestionario aplicado a los clientes evidencia que la mayoría de los contratos de arriendo son para fin comercial y administración para su acreditación solicita información (C.I, RUC, Papeleta de votación). Del 100\% de la cartera el 32.4\% presenta retrasos en el pago de las obligaciones, además el 23.5\% ha tenido inconvenientes con la empresa por falta de pago, cuyo método preferido es el depósito. Además, la mayoría de los arrendatarios ha sido cliente por más de un año resaltando la conformidad con la propiedad y el servicio proporcionado.

\subsection{LEVANTAR INFORMACIÓN ACERCA DEL PROCESO DE CONCESIÓN Y RECUPERACIÓN DE LA CARTERA PARA ESTRUCTURAR UNA MESOLOGÍA DE TRABAJO EFECTIVA}

\section{- $\quad$ Narrativa al proceso de concesión}

El proceso de concesión de crédito inicia por parte del arrendatario, este solicita audiencia con la administradora con el fin de explicar el motivo de la solicitud de concesión. Al acordarse la audiencia el arrendatario se acerca a la empresa para exponer su caso (motivo personal, robo), el cual debe estar respaldado por la documentación respetiva que es específica para cada situación. La administradora analizará esta información para determinar la gravedad del caso y precisar si se debe conceder el crédito, este proceso dura 24 horas y se notificará al cliente al día siguiente. Si el crédito es concedido se establecerá de forma verbal los pazos de pago, si es denegado el cliente deberá presentar información adicional concreta.

\section{- $\quad$ Narrativa al proceso de recuperación}

La recuperación de la cartera inicia con una revisión a la base de datos para identificar qué arrendatario está próximo a saldar su deuda por vencimiento de su cuota, una vez identificado la administradora le notificará por medio de vía telefónica, si el cliente atiende al llamado e indica que se acercara a cancelar la deuda (efectivo, cheque, depósito), la recepción de este pago es recibido por la contadora, quien procede a verificar el monto, actualizar la base de datos, emitir y entregar el respectivo 
comprobante de pago. En el caso de que el cliente no atendiera, la administración insistirá hasta conseguir una respuesta, si el cliente se encuentra atrasado deberá acercarse para solicitar la concesión respectiva.

Si el arrendatario no brinda una solución adecuada o colaboración, la abogada externa elaborará un escrito solicitando la pronta cancelación de la deuda, si de todas formas no hay respuestas, se redactará una citación judicial formal; una vez concluido el proceso judicial respectivo en donde se pactó un convenio formal de pago, el cual si no es cumplido como medida extrema se aplicará un juicio de embargo para recuperar el valor de la cartera de forma parcial o total.

6.3 ELABORAR LOS PROGRAMAS DE TRABAJO PARA REALIZAR EL PROCESO DE AUDITORÍA Y DETERMINAR EL NIVEL DE RIESGO Y CONFIANZA DE LOS PROCESOS A AUDITAR

Referente al segundo objetivo de investigación que detalla la elaboración de planes para las diferentes fases de la auditoría. La primera denominada de planificación básicamente especifica el conjunto de documentos, instrumentos y acercamientos solicitados y aplicados por el grupo de trabajo de forma directa al departamento de administración, con la intención de obtener los primeros datos que brindarían una perspectiva general de la organización en lo que respecta a los procedimientos de concesión y recuperación de la cartera de la empresa Diócesis de Santo Domingo. A continuación, se presenta el programa de planificación atribuible a la primera fase del plan de auditoría.

Tabla 2 Programa de auditoría para la fase de Planificación al proceso de concesión y recuperación de cartera

\begin{tabular}{|c|c|c|c|c|}
\hline \multicolumn{5}{|c|}{$\begin{array}{l}\text { PROGRAMA DE AUDITORÍA PARA LA FASE DE PLANIFICACIÓN, AUDITORÍA DE GESTIÓN AL PROCESO DE CONCESIÓN Y } \\
\text { RECUPERACIÓN DE CARTERA }\end{array}$} \\
\hline $\mathrm{N}^{\circ}{ }^{\circ}$ & Procedimiento & Código & Elaborado por: & Fecha: 1-07-2021 \\
\hline 1. & $\begin{array}{l}\text { FASE 1: } \\
\text { VALORACIÓN SI }\end{array}$ & Cación & LA EMPRESA & \\
\hline 1.1 . & $\begin{array}{l}\text { Agendar una audiencia con la administradora de la } \\
\text { empresa Diócesis de Santo Domingo en Ecuador, para } \\
\text { identificar de forma superficial a través de su } \\
\text { testimonio las deficiencias que presenta el proceso de } \\
\text { concesión y recuperación de la cartera. }\end{array}$ & P.1. & EEOB & $1 / 7 / 2021$ \\
\hline 1.2 . & $\begin{array}{l}\text { Recopilar información de forma ordenada y detallada } \\
\text { de las políticas, funciones, normativa, manuales y } \\
\text { otros documentos de importancia necesarios de la } \\
\text { Diócesis de Santo Domingo. }\end{array}$ & P.2. & KMDM & $3 / 7 / 2021$ \\
\hline 1.3 . & $\begin{array}{l}\text { Identificar la estructura organizacional de la empresa } \\
\text { Diócesis. }\end{array}$ & P.3. & EEOB & $5 / 7 / 2021$ \\
\hline 1.4. & $\begin{array}{l}\text { Determinar políticas y procedimientos del proceso de } \\
\text { concesión y recuperación de la cartera }\end{array}$ & P.4. & KMDM & $5 / 7 / 2021$ \\
\hline 1.5. & $\begin{array}{l}\text { Obtener información sobre el lugar de trabajo } \\
\text { utilizado en la industria, el personal relacionado y la } \\
\text { administración existente: } \\
\text { A) Grado de independencia entre el personal } \\
\text { administrativo y su forma de comunicación. } \\
\text { B) Posibles restricciones de cada cargo }\end{array}$ & P.5. & EEOB & $6 / 7 / 2021$ \\
\hline 1.6. & $\begin{array}{l}\text { Elaborar una narrativa del proceso de concesión de } \\
\text { créditos. }\end{array}$ & P.6. & KMDM & $6 / 7 / 2021$ \\
\hline 1.7. & $\begin{array}{l}\text { Elaborar una narrativa del proceso de recuperación de } \\
\text { la cartera. }\end{array}$ & P.7. & EEOB & 7/7/2021 \\
\hline
\end{tabular}

Nota. Documentación e información solicitada al dpto. de Administración. Fuente: Elaboración propia. 
La segunda fase del programa de auditoría denominada de evaluación se refiere a las acciones realizadas por el grupo de trabajo para identificar los niveles de riesgo y confianza e inclusive la determinación de las posibles áreas críticas existentes en el procedimiento de concesión y recuperación de la cartera cuya función es realizada por el departamento de administración de la empresa. La herramienta principal utilizada en esta segunda fase fue el cuestionario de control interno el cual fue elaborado bajo los estándares determinados por el COSO II ERM facilitando la identificación de las áreas críticas y el nivel de riesgo en que se encuentra la organización que según los resultados es moderado con $63.57 \%$.

Tabla 3 Nivel de confianza y riesgo (Resultados Cuestionario de Control Interno)

EMPRESA DIÓCESIS DE SANTO DOMINGO EN ECUADOR, DEPARTAMENTO
Dragrama Auditorio
DE ADMNISTRACIÓN.

Objetivo: Determinar el nivel de confianza y riesgo del cuestionario de control interno

Relevamiento:

MATRIZ DE CALIFICACIÓN DEL NIVEL DE CONFIANZA

\begin{tabular}{|c|c|c|c|c|c|}
\hline N. ${ }^{\circ}$ & Componente & $\begin{array}{l}\text { Componente } \\
\text { ponderado }\end{array}$ & $\begin{array}{c}\text { Calificación } \\
\text { de la } \\
\text { Ponderación }\end{array}$ & Total & Nivel de confianza \\
\hline 1 & Ambiente de control & $9,14 \%$ & $15,43 \%$ & $59,26 \%$ & Bajo \\
\hline 2 & Definición de objetivos & $3,57 \%$ & $8,29 \%$ & $43,10 \%$ & Moderado \\
\hline 3 & Identificación de riesgos & $16,64 \%$ & $25,71 \%$ & $64,72 \%$ & Moderado \\
\hline 4 & Evaluación de riesgos & $8,14 \%$ & $10,71 \%$ & $76,00 \%$ & Alto \\
\hline 5 & Respuesta al riesgo & $11,86 \%$ & $15,43 \%$ & $76,85 \%$ & Alto \\
\hline 6 & Actividades de control & $2,36 \%$ & $7,86 \%$ & $30,00 \%$ & Bajo \\
\hline 7 & Información y comunicación & $6,71 \%$ & $8,29 \%$ & $81,03 \%$ & Alto \\
\hline 8 & Monitoreo & $5,14 \%$ & $8,29 \%$ & $62,07 \%$ & Moderado \\
\hline & Total & $63,57 \%$ & $100,00 \%$ & $63,57 \%$ & Moderado \\
\hline \multicolumn{6}{|c|}{$\%$ Nivel de Confianza $=\frac{\% \text { Componente Ponderado }}{\% \text { Calificación dePonderación }}$} \\
\hline \multirow{2}{*}{\multicolumn{2}{|c|}{ Nivel de Confianza }} & \multirow[b]{2}{*}{ Resultados } & \multicolumn{2}{|c|}{ Nivel de Riesgo } & \multirow[b]{2}{*}{ Resultado } \\
\hline & & & Escala & Valoración & \\
\hline & $76 \%-95 \%$ & \multirow{3}{*}{$63,57 \%$} & Bajo & 1 & \multirow{3}{*}{40} \\
\hline & $50 \%-75 \%$ & & Moderado & 2 & \\
\hline & $15 \%-49 \%$ & & Alto & 3 & \\
\hline
\end{tabular}

Nota. Resultados componentes Cuestionario de Control interno. Fuente: Elaboración propia.

La tercera fase del plan de auditoria también denominada de ejecución consistió en una corroboración de información en donde el equipo de trabajo con los datos proporcionados por el departamento de administración analizo la veracidad de los datos atravesó de diferentes matrices. En esta 
Tabla 4 Indicadores de Gestión

fase se realizaron seis papeles de trabajo en primero se analizó la veracidad de los cánones de arriendo de los clientes establecidos en la base de datos, el segundo consistió en él envió de cartas de circularización a los arrendatarios, en el tercero se rectificaban las posibles inconsistencias encontradas, en el cuarto se aplicaron diferentes indicadores de gestión sobre diferentes aspectos del departamento y los dos últimos se centraron en el cumplimiento de políticas y veracidad del archivo físico.

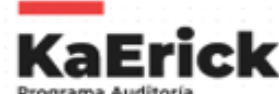

EMPRESA DIÓCESIS DE SANTO DOMINGO EN ECUADOR, DEPARTAMENTO DE ADMNISTRACIÓN.

Objetivo: Auditar el saldo del rubro cuentas por cobrar en referencia a las respuestas de los arrendatarios a las cartas de circularización y realizar los respectivos ajustes en caso de ser necesario.

Resumen resultados de indicadores

\begin{tabular}{|c|c|c|c|c|c|}
\hline N. ${ }^{\circ}$ & Indicador & Fórmula & Resultado E. & Proceso & Análisis \\
\hline 1 & $\begin{array}{l}\text { N. }{ }^{\circ} \text { de politicas } \\
\text { cumplidas en el } \\
\text { proceso de } \\
\text { concesión de } \\
\text { créditos }\end{array}$ & $\begin{array}{l}\mathrm{N}{ }^{\circ} \text { Politicas de } \\
\text { concesión de } \\
\text { créditos } \\
\text { cumplidas / Total } \\
\text { de políticas de } \\
\text { concesión } \\
\text { establecidas }\end{array}$ & $\begin{array}{l}\text { El resultado del } \\
\text { indicador debe ser } \\
100 \%\end{array}$ & $100 \%$ & $\begin{array}{l}\text { Del total de las políticas establecidas en } \\
\text { el área de concesión de créditos el } \\
100 \% \text { representa un cumplimiento total } \\
\text { de las politicas por parte del } \\
\text { departamento de administración }\end{array}$ \\
\hline 2 & $\begin{array}{l}\mathrm{N} \cdot \text { de empleados } \\
\text { del departamento } \\
\text { de } \\
\text { administración }\end{array}$ & $\begin{array}{l}\text { N. Empleados } \\
\text { capacitados } \\
\text { (proceso de } \\
\text { concesion) / Total }\end{array}$ & $\begin{array}{l}\text { E1 resultado del } \\
\text { indicador debe ser } \\
\qquad 100 \%\end{array}$ & $\frac{0}{2}$ & $\begin{array}{l}\text { Del total de empleados capacitados que } \\
\text { pertenecen al departamento de } \\
\text { administración el } 0 \% \text { significa que los } \\
\text { empleados que integran este }\end{array}$ \\
\hline & $\begin{array}{l}\text { capacitados en el } \\
\text { proceso de } \\
\text { concesión }\end{array}$ & $\begin{array}{l}\text { empleados Dep. } \\
\text { Administración }\end{array}$ & & & $\begin{array}{c}\text { departamento no son capacitados en el } \\
\text { área de concesión de créditos }\end{array}$ \\
\hline 3 & $\begin{array}{l}\mathrm{N} \cdot{ }^{\circ} \text { de politicas } \\
\text { cumplidas en el } \\
\text { proceso de } \\
\text { recuperación de } \\
\text { la cartera }\end{array}$ & $\begin{array}{l}\text { N. Politicas de } \\
\text { recuperación de } \\
\text { cartera cumplidas } \\
\text { / Total de } \\
\text { politicas de } \\
\text { recuperación } \\
\text { establecidas }\end{array}$ & $\begin{array}{l}\text { E1 resultado del } \\
\text { indicador debe ser } \\
100 \%\end{array}$ & $100 \%$ & $\begin{array}{l}\text { Del total de las políticas establecidas en } \\
\text { el área de recuperación de la cartera el } \\
100 \% \text { representa un cumplimiento total } \\
\text { de las políticas por parte del } \\
\text { departamento de administración }\end{array}$ \\
\hline 4 & $\begin{array}{l}\text { N. de empleados } \\
\text { del departamento } \\
\text { de } \\
\text { administración } \\
\text { capacitados en el } \\
\text { proceso de } \\
\text { recuperación de } \\
\text { cartera }\end{array}$ & $\begin{array}{c}\text { N. Empleados } \\
\text { capacitados } \\
\text { (proceso de } \\
\text { recuperación) } / \\
\text { Total empleados } \\
\text { Dep. } \\
\text { Administración }\end{array}$ & $\begin{array}{l}\text { E1 resultado del } \\
\text { indicador debe ser } \\
\qquad 100 \%\end{array}$ & $\frac{0}{2}$ & $\begin{array}{l}\text { Del total de empleados capacitados que } \\
\text { pertenecen al departamento de } \\
\text { administración el } 0 \% \text { significa que los } \\
\text { empleados que integran este } \\
\text { departamento no son capacitados en los } \\
\text { procesos de recuperación de cartera }\end{array}$ \\
\hline
\end{tabular}




\begin{tabular}{|c|c|c|c|c|c|}
\hline 5 & $\begin{array}{c}\text { Nivel de } \\
\text { morosidad de la } \\
\text { cartera }\end{array}$ & $\begin{array}{l}\mathrm{N} \cdot{ }^{\circ} \text { clientes con } \\
\text { retrasos en sus } \\
\text { pagos / Clientes } \\
\text { totales }\end{array}$ & $\begin{array}{c}\mathrm{E} 1 \text { indicador debe } \\
\text { estar entre } 0 \% \text { a } \\
10 \%\end{array}$ & $\begin{array}{l}\frac{8}{34} \\
24 \%\end{array}$ & $\begin{array}{l}\text { E1 nivel de morosidad de la cartera es } \\
\text { igual a un } 24 \% \text { valor que supera de } \\
\text { forma considerable el intervalo de } \\
\text { morosidad aceptado por la empresa }\end{array}$ \\
\hline 6 & $\begin{array}{l}\text { Nivel de } \\
\text { recuperación de } \\
\text { las cuentas } \\
\text { incobrables }\end{array}$ & $\begin{array}{c}\text { Monto } \\
\text { recuperado / } \\
\text { Total deuda }\end{array}$ & $\begin{array}{l}\text { E1 indicador debe } \\
\text { dar } 95 \% \text { a } 100 \%\end{array}$ & $\frac{90.911,02}{114.441,16}$ & $\begin{array}{c}\text { E1 nivel de recuperación de las cuentas } \\
\text { incobrables es igual a un } 79,44 \%, \\
\text { porcentaje que no figura dentro del } \\
\text { intervalo aceptado por empresa } \\
\text { Diócesis }\end{array}$ \\
\hline 7 & $\begin{array}{l}\mathrm{N} .{ }^{\circ} \text { pagos } \\
\text { realizados de } \\
\text { forma parcial }\end{array}$ & $\begin{array}{l}\mathrm{N} .{ }^{\circ} \text { clientes que } \\
\text { realizan pagos } \\
\text { forma parcial } / \\
\text { Total clientes }\end{array}$ & $\begin{array}{c}\text { E1 resultado del } \\
\text { indicador debe ser } \\
0 \%\end{array}$ & $\begin{array}{c}\frac{10}{34} \\
29,41 \%\end{array}$ & $\begin{array}{l}\text { De la totalidad de pagos realizados por } \\
\text { los arrendatarios el } 29,41 \% \text { representa } \\
\text { el porcentaje de clientes que realizan } \\
\text { abonos y no saldan en su totalidad el } \\
\text { canon de arriendo mensual }\end{array}$ \\
\hline 8 & $\begin{array}{c}\% \text { de asientos } \\
\text { contables con } \\
\text { errores (mensual) }\end{array}$ & $\begin{array}{l}\mathrm{N} .^{\circ} \text { asientos } \\
\text { erróneos / total } \\
\text { asientos }\end{array}$ & $\begin{array}{l}\text { E1 resultado del } \\
\text { indicador debe } \\
\text { estar entre } 0 \% \text { a } \\
5 \%\end{array}$ & $\frac{0}{44}$ & $\begin{array}{l}\text { Del total de asientos realizados en el } \\
\text { mes de diciembre por concepto de } \\
\text { arriendos el } 0 \% \text { significa que no existen } \\
\text { asientos con errores dentro del archivo } \\
\text { y el sistema contable }\end{array}$ \\
\hline & $\begin{array}{l}\% \text { de ingresos } \\
\text { que representa el } \\
\text { arriendo de } \\
\text { bienes inmuebles } \\
\text { para la empresa }\end{array}$ & $\begin{array}{c}\text { Total, ingresos } \\
\text { por arriendo de } \\
\text { bienes inmuebles } \\
\text { / Total ingresos } \\
\text { percibidos por la } \\
\text { empresa }\end{array}$ & $\begin{array}{l}\text { E1 resultado del } \\
\text { indicador debe } \\
\text { estar entre } 10 \% \text { a } \\
20 \%\end{array}$ & $\frac{126.290,36}{1^{\prime} 226,418,18}$ & $\begin{array}{l}\text { E1 nivel de ingresos por consecuencia } \\
\text { de arriendos representa un } 10,30 \% \text { de } \\
\text { los ingresos totales percibidos por la } \\
\text { empresa Diócesis }\end{array}$ \\
\hline
\end{tabular}

Nota. Aplicación de indicadores de gestión en aspectos referentes al proceso de concesión y recuperación de cartera. Fuente: Elaboración propia.

\subsection{ELABORACIÓN DEL INFORME DE AUDITORÍA}

El informe de auditoría redactado como culminación del examen al proceso de concesión y recuperación de la cartera de la empresa Diócesis de Santo Domingo en Ecuador a través de una auditoría de gestión, se realizó con el objetivo de valorar la eficiencia, eficacia, efectividad, ética y otros aspectos en el proceso mencionado anteriormente, donde a través de diferentes metodologías y actividades específicas diseñadas para este tipo de auditoría se identificaron ciertas deficiencias como: la ausencia de un manual en donde consten detalladas políticas y procedimientos de forma escrita que guíen el accionar del departamento en lo referente a créditos y cobranzas.

Otro aspecto importante a considerar son la inexistencia de criterios de cumplimiento determinados para el nivel de morosidad de la cartera, nivel de deterioro de las cuentas por cobrar y el monto recuperado aceptable, estos y otros aspectos se encuentran detallados en el informe de auditoría en donde además de la conclusión respectiva por cada área crítica identificada se proporcionó una recomendación que debe considerarse como una medida preventiva, a las cuáles se recomienda dar 
seguimiento en un periodo no superior a seis meses con el propósito de monitorear si existen indicios de mejora.

\section{DISCUSIÓN}

La recopilación de información empezó con visitas a las instalaciones, por medio de entrevistas aplicadas a los empleados que integran el departamento administrativo, encargado de los créditos y las cobranzas, proporcionando una perspectiva general de la situación empresarial actual e identificando la problemática y los posibles puntos de interés a considerar en el examen de auditoría. En el continuo proceso de levantamiento de información se pudo notar la ausencia de un manual de políticas y funciones que detalla de forma específica cómo debe proceder el empleado al momento de conceder un crédito, las variables a considerar (retraso, monto, motivo), inconvenientes que se presentan de igual forma en la recuperación de la cartera.

En la aplicación del cuestionario de control interno COSO II ERM se determinó que el nivel de confianza es moderado siendo este igual a $63,57 \%$ determinando una muestra del $40 \%$ de nuestra población total integrada por los 34 clientes considerados para este estudio, la muestra utilizada estaba compuesta por 14 clientes, seleccionados por medio de la modalidad de conveniencia para asegurar la veracidad de los resultados. Además de que se identificaron los componentes de control interno con un nivel de confianza bajo que son el ambiente de control con $59.26 \%$ y las actividades de control con $30 \%$, coeficientes que de cierta forma dan una explicación a los resultados de los indicadores.

La fase tres del plan de auditoría inicio con una verificación de los saldos de los 34 clientes que integran la población, acción que facilitó la determinación de la muestra en cuya selección se consideró los abonos realizados, la cantidad de meses de retraso y el saldo pendiente de pago, una vez realizado este procedimiento se procedió a redactar la respectiva carta de circularización a los 14 integrantes de la muestra seleccionados con los criterios detallados anteriormente. Una vez obtenida la respuesta de las cartas de circularización por parte de los clientes se analizaron para identificar si existía inconformidad con los saldos o la necesidad de un ajuste en la información recopilada.

\section{CONCLUSIONES Y RECOMENDACIONES}

La aplicación de los diferentes instrumentos de recogida de datos (entrevista -encuesta) al departamento de administración y a los clientes de la Diócesis de Santo Domingo proporcionaron una perspectiva general del proceder y la situación actual de la organización, además de una guía que facilitó la identificación de varios puntos de interés a considerar para la elaboración de trabajo. Una de las principales deficiencias observadas es la ausencia de un manual de políticas y funciones además de un 
perfil de cargos definidos para el puesto del jefe del departamento y el contador por lo que consideramos conveniente su elaboración específica acorde a las necesidades del departamento.

A través de la aplicación del Cuestionario de Control Interno COSO II ERM instrumento elaborado específicamente para determinar el nivel confianza de la organización en el área de créditos y cobranzas, cuyo nivel de riesgo fue moderado con un resultado de 63,57\%; además de los componentes de control interno con un nivel de riesgo alto que son el ambiente de control y las actividades de control. Aunque el nivel de confianza sea moderado sería recomendable que la empresa establezca estándares de cumplimiento específicos, además de una detallada distribución de funciones para cuyo propósito se sugiere la creación de un manual de políticas y procedimientos en donde es preciso que se detallen también las restricciones.

La fase de evaluación evidenció la existencia de ciertas deficiencias presentes en el procedimiento de créditos y cobranzas principalmente en lo referente a la recuperación de la cartera, pues se encontraron situaciones donde los clientes poseen un periodo de retraso superior a los 12 meses, además de resaltar la ausencia de estándares o criterios de cumplimiento que indiquen los rangos aceptables de morosidad de cartera, saldos recuperados y deterioro de la misma. Se recomienda que de forma semestral se reúnan el contador, el jefe del departamento y el Sr Obispo para la creación de estándares o modificarlos según la necesidad presentada.

En el informe de auditoría al proceso de concesión y recuperación de la cartera a la empresa Diócesis de Santo Domingo en Ecuador se redactó con la intención de comunicar todos los hallazgos y hechos relevantes realizados por el grupo de trabajo a lo largo del proceso de auditoría, dando a conocer a través de una síntesis los aspectos más relevantes en los cuales se identificó el posible riesgo. Se recomienda a la empresa considerar y aplicar las recomendaciones proporcionadas con la finalidad de corregir deficiencias identificadas en el proceso de auditoría que además de una medida correctiva estas pueden ser utilizadas para anticipar futuros inconvenientes que puedan afectar la eficiencia y eficacia del procedimiento. 


\section{REFERENCIAS}

Alcívar-Cedeño, F. M., Brito-Ochoa, M. P., \& Guerrero-Carrasco, M. J. (Julio-Septiembre de 2016). AUDITORÍA EN LAS EMPRESAS. Contribuciones a la Economía, 1-15. Recuperado el 26 de Enero de 2021, de https://www.eumed.net/ce/2016/3/auditoria.html

Arias-Gómez, J., Villasis-Keever, M. Á., \& Miranda-Novales, M. G. (2 de Abril-Junio de 2016). El protocolo de investigación III: la población de estudio. Revista Alergia México, LXIII(2), 201-206. Recuperado el 10 de Febrero de 2021, de https://www.redalyc.org/pdf/4867/486755023011.pdf

Arias-Gonzáles, I. P. (Abril de 2018). AUDITORÍA UN ENFOQUE DE GESTIÓN. Observatorio de la Economía Latinoamericana, 1-6. Recuperado el 27 de Enero de 2021, de https://www.eumed.net/rev/oel/2018/04/auditoria-gestion.html

Betancur-Marín, J. C., \& Acosta-Santamaría, D. A. (2014). ¿CÓMO SE SOSTIENEN FINANCIERAMENTE LAS EMPRESA SIN ÁNIMO DE LUCRO? CASOS DE ESTUDIO EN EL ÁREA METROPOLITANA DE BUCARAMANGA. Universidad Industrila de Santander, Facultad de Ingenierías Físiomecánicas. Bucaramanga: Universidad Industrila de Santander. Recuperado el 16 de Diciembre de 2021, de http://tangara.uis.edu.co/biblioweb/tesis/2014/155762.pdf

Biler-Reyes, S. A. (6 de Marzo de 2017). Auditoria. Elementos esenciales. Ciencias económicas y empresariales, III(3), 138-151. doi:10.23857/dc.v3i1.379

Campos-Muñiz, A., Castañeda-Audiffred, R., Holguín-Maillard, F., López-Prado, A., \& Tejero-Gonzáles, A. (2018). Auditoría de estados financieros y su documentación. Con énfasis en riesgos (Primera ed.). Ciudad de México, México: Instituto Méxicano de Contadores Públicos. Recuperado el 31 de Enero de 2021, de https://books.google.es/books?hl=es\&lr=\&id=Eb1XDwAAQBAJ\&oi=fnd\&pg=PT6\&dq=papeles+de+trabaj o\&ots=aGE4zRQrap\&sig=DfdgQvIs6M5O4RatOfZnQlZExSg\#v=onepage\&q=papeles\%20de\%20trabajo\&f $=$ false

Carrión, H., Mendoza, M., \& Vera, C. (2 de Mayo de 2017). Importancia de la auditoría interna para el perfeccionamiento de los niveles eficiencia y calidad en las empresas. Domino de las Ciencias, III(2), 908920. doi:10.23857

Cubero-Abril, T. (2019). Manual deauditoría de gestión Enfoque empresarial y de riesgos. Universidad del Azuay, Facultad de Ciencias de la Administración. Azuay: Casa Editora. Recuperado el 27 de Enero de 2021, de http://publicaciones.uazuay.edu.ec/index.php/ceuazuay/catalog/view/82/76/674-1

Cuenca-Zapata, E. E. (2017). Análisis del proceso de cartera vencida en el negocio “Almacenes Derick" en el periodo 2015-2016. Universidad Católica de Cuenca Extensión San Pablo de La Troncal, Ciencias Administrativas y Contables. Cuenca: Universidad Católica de Cuenca. Recuperado el 19 de Enero de 2021, de

http://186.5.103.99/bitstream/reducacue/7643/1/TRABAJO\%20DE\%20TITULACION\%20ERIKA\%20CUE NCA.pdf

Díaz-Bravo, L., Torruco-García, U., Martínez-Hernández, M., \& Varela-Ruiz, M. (Julio-Septiembre de 2013). La entrevista, recurso flexible y dinámico. Investigación en educación Médica, 162-167. Recuperado el 25 de Febrero de 2021, de https://www.redalyc.org/pdf/3497/349733228009.pdf

Label, W., Ledesma, J. d., \& Ramos-Arriaga, R. A. (2016). Contabilidad para no contadores : una forma rápida y sencilla de entender la contabilidad (Segunda ed.). Bogotá, Colombia: Ecoe Ediciones. Recuperado el 2021 de 31 de Enero, de https://bibliotecavirtual.puce.edu.ec/reader/contabilidad-para-no-contadores-labelledesma-ramos?location $=4$ 
Landes-Foster, W., Kim, P., \& Cristiansen, B. (3 de Marzo de 2021). Diez modelos de financiamiento para organizaciones sin fines de lucro. Stanford Social Innavation, 1-5. Recuperado el 26 de DIciembre de 2021, de https://ssires.tec.mx/es/noticia/diez-modelos-de-financiamiento-para-organizaciones-sin-fines-de-lucro

Lopez, H. (26 de Agosto de 2016). UNIVIA. Recuperado el 27 de Enero de 2021, de http://univia.info/politicas-de-cobranza/

López-Roldan, P., \& Fachelli, S. (2015). Metodología de la Investigación social cuantitativa (Primera ed.). Barcelona, España: Universidad Autónoma de Barcelona. Recuperado el 10 de Febrero de 2021, de https://ddd.uab.cat/pub/caplli/2016/163567/metinvsoccua_a2016_cap2-3.pdf

Martínez, F., Ramírez, S., Montoya del Corte, J., \& Fernández, A. (2010). La importancia relativa en auditoría. Las nuevas normas internacionales en comparación con la normativa vigente en Iberoamérica. UNAM, Facultad de Contaduría y Administración. Mèxico: Contadurìa y administraciòn. Recuperado el 14 de Diciembre de 2020, de http://www.scielo.org.mx/scielo.php?script=sci_arttext\&pid=S018610422010000100005

Montes de Oca, J. (20 de Julio de 2015). Economipedia. Recuperado el 25 de Febrero de 2021, de https://economipedia.com/definiciones/credito.html

Prado, J., Erráez, J., Cilio, I., Godoy, D., \& Nicole, G. (2019). La Banca en el Ecuador: Un Enfoque Provincial. ASOBANCA, Departamento Económico. Quito: ASOBANCA. Recuperado el 26 de Diciembre de 2020, de https://asobanca.org.ec/wp-content/uploads/2021/07/La-Banca-en-Ecuador-Un-Enfoque-Provincial-2018Mar-2019.pdf

Quintanilla-Romero, M. A., \& Trujillo-Calero, G. E. (2015). La Gestión en la Auditoría (Primera ed.). Quito, Pichincha, Ecuador: Quintanilla Romero, Marco Antonio. Recuperado el 12 de Febrero de 2021, de http://www.dspace.uce.edu.ec/bitstream/25000/14177/1/La\%20gesti\%C3\%B3n\%20en\%20la\%20auditor\%C $3 \%$ ADa.pdf

Romo-Veloz, M. A. (2016). PROPUESTA DE DISEÑO DE IMPLEMENTACIÓN DE UN MANUAL DE CONTROL INTERNO EN EL MANEJO DE CRÉDITOS Y COBRANZAS PARA LA EMPRESA DIFILCOM. PONTIFICIA UNIVERSIDAD CATÓLICA DEL ECUADOR, FACULTAD DE CIENCIAS ADMINISTRATIVAS Y CONTABLES. Quito: PONTIFICIA UNIVERSIDAD CATÓLICA DEL ECUADOR. Recuperado el 19 de ENERO de 2021, de http://repositorio.puce.edu.ec/bitstream/handle/22000/12318/Tesis-RomoM.pdf?sequence=1\&isAllowed=y

Sánchez-Carlessi, H., Reyes-Romero, C., \& Mejía-Sáenz, K. (2018). Manual de términos en investigación científica, tecnológica y humanística (Primera ed.). (U. R. Palma, Ed.) Lima, Peru: Universidad Ricardo Palma. Recuperado el 12 de Febrero de 2021, de https://www.urp.edu.pe/pdf/id/13350/n/libro-manual-de-terminosen-investigacion.pdf

Tapia, C., Mendoza, S., Castillo, S., \& Guevara, E. (2019). Funadamentos de auditoría. Aplicación prática de las Normas Internacionales de Auditoría (Primera ed., Vol. I). (A. García, \& N. Berenice, Edits.) Ciudad de México, México: Instituto Mexicano de Contadores Públicos. Recuperado el 24 de Enero de 2021, de https://books.google.es/books?id=4TLfDwAAQBAJ\&pg=PT40\&dq=antecedentes+de+la+auditor\%C3\%AD $\mathrm{a} \& \mathrm{lr}=\& \mathrm{hl}=\mathrm{es} \&$ source=gbs_selected_pages\&cad=2\#v=onepage $\& \mathrm{q}=$ antecedentes $\% 20 \mathrm{de} \% 201 \mathrm{a} \% 20$ auditor $\%$ $\mathrm{C} 3 \% \mathrm{ADa} \& \mathrm{f}=$ false

Ulloa-Zuñiga, G. F. (2020). ANÁLISIS DEL NIVEL DE MOROSIDAD DE LA CARTERA QUE MANTIENE LA COOPERATIVA DE AHORRO Y CRÉDITO FASAYÑAN". Universidad Politécnica Saleciana, Carrera de Administración de empresas. Cuenca: Universidad Politécnica Saleciana. Recuperado el 18 de Enero de 2021, de https://dspace.ups.edu.ec/bitstream/123456789/19427/1/UPS-CT008873.pdf 